\title{
Domain Wall Motion by the Magnonic Spin Seebeck Effect
}

\author{
D. Hinzke and U. Nowak \\ Fachbereich Physik, Universität Konstanz, D-78457 Konstanz, Germany
}

(Received 9 March 2011; published 8 July 2011)

\begin{abstract}
The recently discovered spin Seebeck effect refers to a spin current induced by a temperature gradient in a ferromagnetic material. It combines spin degrees of freedom with caloric properties, opening the door for the invention of new, spin caloritronic devices. Using spin model simulations as well as an innovative, multiscale micromagnetic framework we show that magnonic spin currents caused by temperature gradients lead to spin transfer torque effects, which can drag a domain wall in a ferromagnetic nanostructure towards the hotter part of the wire. This effect opens new perspectives for the control and manipulation of domain structures.
\end{abstract}

DOI: 10.1103/PhysRevLett.107.027205

PACS numbers: 75.76.+j, 75.30.Sg, 75.60.Ch, 75.75.-c

The occurrence of spin currents or spin accumulation in a ferromagnet due to a temperature gradient is called spin Seebeck effect (SSE). The existence of this novel effect was recently demonstrated in thin-film samples of a metallic ferromagnet [1]. The authors suggested that, due to spin-dependent chemical potentials in the ferromagnet, the spin-up and spin-down electrons diffuse to opposite ends of the sample which was placed in a temperature gradient. This spin-dependent diffusion would then create a position-dependent spin accumulation, which was detected via the inverse spin Hall effect. Later on it was shown that the SSE is not limited to metals but occurs in dilute magnetic semiconductors [2] and even in insulators [3]. The existence of the SSE in insulators clearly shows that no electron transport is necessary for its occurrence [4]. Though the details of its origin and how to describe it theoretically remain unclear it is obvious that the SSE can be expected to lead to the development of new spin caloritronic devices, combining the spin degrees of freedom with caloric properties.

Theoretical descriptions of the SSE are mostly based on the transport of heat by diffusive motion of conduction electron in a ferromagnetic metal $[5,6]$. But literally a spin current is a current of angular momentum and not necessarily connected to a charge current. Magnons do also carry angular momentum and, hence, a spin current can be solely magnonic $[4,7]$. Obviously, only a magnonic spin current can possibly explain the experimental observation of a SSE in insulators [3]. Even in a metal a magnonic SSE must appear in addition to charge-based currents since magnons, thermally excited in the hotter region, must always diffusively move towards the colder region. Hence in a ferromagnetic metal one can in principle expect both types of Seebeck effects, the temperature gradient leading to a charge-based spin current as well as a magnonic spin current.

Furthermore, only the magnonic SSE can explain experiments where the nanostructure was cut, with that blocking any possible charge current, but the spin accumulation remained unaltered [2]. Hence, we expect magnons to play an important role for the understanding of the SSE, though much less attention has been paid so far to the theory of magnonic spin currents [8,9]. Even for the case of a metal they might be relevant since so far it is not clear how big the two contributions, charge-based and magnonic, to the SSE are. It is clear, however, that only magnonic spin currents can exist in an insulator.

Charge-based spin-polarized currents following from electrical fields in a spin-polarized material are known to lead to spin transfer torque (STT) effects, that can be exploited for the control of domain wall (DW) motion [10-12]. The existence of considerable STT following from spin currents due to the SSE have been forecast accordingly $[9,13]$. For the case of a single DW in a nanowire this torque should in principle lead to DW motion caused — via the SSE—by temperature gradients.

In the following we will demonstrate the existence of thermally driven DW motion caused solely by magnonic spin currents. Neglecting electron currents our model will be able to describe insulators and a theoretical description of the magnetic degrees of freedom (without any coupling to electron currents) must be sufficient. In the spirit of a multiscale modeling approach [14] we present two different views on thermally induced DW motion, namely, an atomistic approach resting on the stochastic LandauLifshitz-Gilbert (LLG) equation of motion for an atomistic spin model and a micromagnetic approach via the LandauLifshitz-Bloch (LLB) equation of motion, which has been proven over the last decade to link successfully micromagnetic and thermodynamic properties of ferromagnets.

To illustrate the basic concept let us start discussing the behavior of a single DW in a nanowire. We assume the DW to be transverse with a $0 \mathrm{~K} \mathrm{DW}$ width given by $\delta=$ $\pi \sqrt{A / K}$ where $A$ is the exchange stiffness and $K$ the uniaxial anisotropy constant. For finite temperature its fundamental thermodynamic potential is the free energy density $\Delta F(T)=\Delta E-T \Delta S$, where $\Delta E$ is the internal energy density and $\Delta S$ the entropy density of the DW. In the $0 \mathrm{~K}$ limit it approaches the well-known micromagnetic 
expression $\Delta F(T=0)=2 \sqrt{A K}$. For finite temperature $\Delta F(T)$ is a monotonically decaying function of temperature [see Fig. 1(a)] vanishing at the Curie temperature $T_{\mathrm{C}}$ [15]. To minimize its free energy the DW must move towards regions with higher temperatures. Assuming a temperature gradient $d T / d x$ along the space coordinate $x$ of the wire a thermal pressure (force density) $p=-(d F / d T)(d T / d x)$ will act on the DW. Following Ref. [15] a typical temperature gradient of a DW free energy density is of the order of $2 \times 10^{-5} \mathrm{~J} / \mathrm{m}^{2} \mathrm{~K}$. In modern Laser experiments temperature gradients can be of the order of $1000 \mathrm{~K} / \mu \mathrm{m}$ which would create a pressure of $2 \times 10^{5} \mathrm{~N} / \mathrm{m}^{2}$. This corresponds to a Zeeman pressure $2 M B$ of a field of roughly $5 \mathrm{mT}$ for a material with a magnetization $M$ of $2 \times 10^{6} \mathrm{~A} / \mathrm{m}$. This effect should clearly be measurable.

However, these purely thermodynamic considerations assume local equilibrium. Though magnons do implicitly contribute to the temperature dependence of the free energy (and all other magnetic properties) a flow of magnons, which is a nonequilibrium phenomenon, is not explicitly taken into account. From a more microscopic viewpoint thermal excitations are explicitly described in terms of spin waves or magnons. As shown in Fig. 1(b) the hotter region of a nanowire would contain a higher density of magnons, which by diffusive motion move into the cooler region. Assuming conservation of angular momentum, the
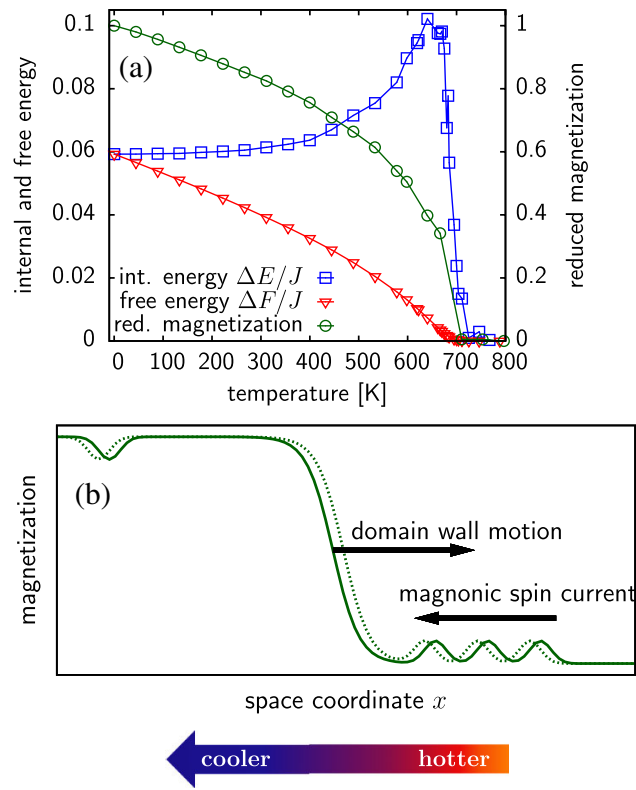

FIG. 1 (color online). (a) Temperature dependence of the free energy $\Delta F / J$ and the internal energy $\Delta E / J$ of a DW in a high anisotropic ferromagnet from spin model simulations [15] where $J$ is the sum over all exchange integrals for a single atomic spin. The reduced magnetization is also shown for comparison. The $T_{\mathrm{C}}$ is about $700 \mathrm{~K}$. (b) Sketch illustrating the magnon current through a DW leading to DW motion when conservation of angular momentum is assumed. magnonic spin current drags the DW towards the hotter region. Note that the two pictures do indeed complement each other but only the microscopic model would allow for a calculation of nonequilibrium properties, while the thermodynamic picture at least locally assumes the existence of a free energy - an equilibrium property.

In the following, we begin our numerical investigation in terms of a spin model which is naturally capable of describing spin waves. We consider a highly anisotropic material with a well-localized DW. The details of the Hamiltonian, which was originally derived to describe the magnetic properties of a highly anisotropic material, are described in Ref. [16]. Most importantly, this Hamiltonian describes a generic ferromagnetic material with a $T_{\mathrm{C}}$ of about $700 \mathrm{~K}$ and a large uniaxial anisotropy, leading to well-localized DWs with a DW width of about $4 \mathrm{~nm}$.

In these simulations, we use Langevin dynamics, i.e., simulations of the stochastic LLG equation of motion,

$$
\dot{\mathbf{S}}_{i}=-\frac{\gamma}{\left(1+\lambda^{2}\right) \mu_{\mathrm{s}}}\left(\mathbf{S}_{i} \times \mathbf{H}_{i}+\lambda \mathbf{S}_{i} \times\left(\mathbf{S}_{i} \times \mathbf{H}_{i}\right)\right),
$$

for unit vectors $\mathbf{S}_{i}$, which represent the normalized magnetic moment of an atomic unit cell in the classical limit. $\mu_{\mathrm{s}}$ is the magnitude of that magnetic moment and assumed temperature independent. The internal field is $\mathbf{H}_{i}=-\frac{\partial \mathcal{H}}{\partial \mathbf{S}_{i}}+\boldsymbol{\zeta}_{i}(t)$ and thermal fluctuations are included here as an additional white-noise term $\zeta_{i}(t) . \gamma$ is the absolute value of the gyromagnetic ratio and $\lambda$ is the microscopic damping constant, which describes the coupling of the spin system to the heat bath phenomenologically. The heat bath is provided by the electronic degrees of freedom as well as by the lattice and it defines the temperature in the canonical ensemble [17].

We model a nanowire of size of $128 \times 16 \times 16$ unit cells of an fcc lattice with lattice constant $0.38 \mathrm{~nm}$. By fixed, antiparallel boundary conditions a DW is forced into the system. Along the long axis $(x)$ we apply a given temperature gradient. Initially the DW is positioned in the middle of the nanowire and the DW profile is transverse in the $x z$ plane. Figure 2 shows the DW at different times for a

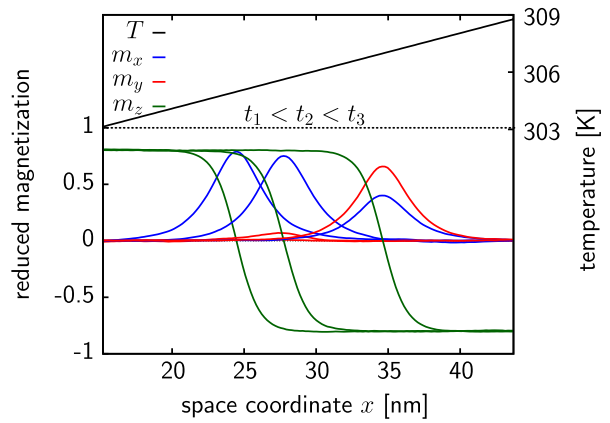

FIG. 2 (color online). All three magnetization components of the DW profiles at different times $\left(t_{1}=0.0 \mathrm{~ns}, t_{2}=0.5 \mathrm{~ns}\right.$, and $\left.t_{3}=1 \mathrm{~ns}\right) . \lambda=0.1$ and $d T / d x=0.197 \mathrm{~K} / \mathrm{nm}$. 
temperature gradient of $d T / d x=0.197 \mathrm{~K} / \mathrm{nm}$. As one can see the DW moves towards the hotter end of the wire and its shape changes slightly due to the fact that the magnetization shrinks approaching the hotter region. Note that the DW motion here is accompanied by precession.

As we have already argued, this type of DW motion has two different origins: on the one hand the temperature dependent change of the free energy of the DW and, on the other hand, the continuous stream of magnons passing the DW. The action of the magnons can be separated from the thermodynamic aspects in simulations where the temperature is constant (e.g., zero) in that part of the system, which contains the DW, and magnons are injected from a distant, hotter region, which acts as a magnon source. In this case our simulations (not presented here) do indeed indicate a domain motion as well, though the thermodynamic equilibrium properties of the DW do not vary during its motion. Magnon diffusion lengths have been reported to be up to $1 \mathrm{~mm}$ [8] in materials with low damping so that normally one can expect magnons to pass DWs easily. On the other hand, the flow of magnons can be interrupted in the high damping limit where the diffusion length of the magnons is limited to below the DW width. In this limit temperature gradient driven DW motion is only due to the spatial variation of the free energy and can be simulated using an innovative micromagnetic framework.

Furthermore, for atomic resolution a DW is already a rather large object and one has to follow that moving object for up to several nanoseconds to obtain an average velocity. Because spin model simulations are much too demanding in terms of computation power, a systematic investigation of the DW motion, its velocity, and its mechanism can hardly be achieved. Here, a micromagnetic framework resting on a continuum model for the magnetization is needed. Conventional micromagnetics, resting on the LLG equation of motion, is a low temperature approach and certainly not valid in this case where temperature gradients dominate the physical behavior of the system. However, an improved framework for thermal micromagnetic calculations was recently developed [14]. It rests on the LLB equation of motion [18] for a thermally averaged spin polarization $\mathbf{m}_{\mathbf{i}}$. This equation of motion does not conserve the length of the magnetization vector, which here represents a thermal average of the fluctuating spin variable $\mathbf{S}_{i}$ of Eq. (1). It rather allows for longitudinal fluctuations of the magnetization in space and time and all relevant magnetic material parameters become functions of temperature. The LLB equation of motion reads

$$
\begin{aligned}
\dot{\mathbf{m}}_{i}= & -\gamma \mathbf{m}_{i} \times \mathbf{H}_{\mathrm{eff}}^{i}+\frac{\gamma \alpha_{\|}}{m_{i}^{2}}\left(\mathbf{m}_{i} \cdot \mathbf{H}_{\mathrm{eff}}^{i}\right) \mathbf{m}_{i} \\
& -\frac{\gamma \alpha_{\perp}}{m_{i}^{2}} \mathbf{m}_{i} \times\left(\mathbf{m}_{i} \times \mathbf{H}_{\mathrm{eff}}^{i}\right) .
\end{aligned}
$$

Besides the usual precession and relaxation terms, the LLB equation contains another term which controls longitudinal relaxation. Here, $\alpha_{\|}$and $\alpha_{\perp}$ are dimensionless longitudinal and transverse damping parameters that are weakly temperature dependent [14]. The effective fields $\mathbf{H}_{\text {eff }}$ are given by

$$
\begin{aligned}
\mathbf{H}_{\mathrm{eff}}^{i}= & \frac{m_{x}^{i} \mathbf{e}_{x}+m_{y}^{i} \mathbf{e}_{y}}{\tilde{\chi}_{\perp}}-\frac{2 A}{m_{\mathrm{e}}^{2} M_{\mathrm{s}}^{0} \Delta^{2}} \sum_{j}\left(\mathbf{m}_{j}-\mathbf{m}_{i}\right) \\
& +\frac{1}{2 \tilde{\chi}_{\|}}\left(1-\frac{m_{i}^{2}}{m_{\mathrm{e}}^{2}}\right) \mathbf{m}_{i} .
\end{aligned}
$$

Here, the first term represents the anisotropy field which makes the $z$ axis the easy axis of the model. The second term is the exchange field where $M_{s}^{0}$ is the zerotemperature saturation magnetization and $\Delta$ is the cell size of the mesh. The thermodynamic equilibrium input functions such as equilibrium magnetization $m_{e}(T)$, exchange stiffness $A(T)$, and susceptibilities $\tilde{\chi}_{\|}(T)$ and $\tilde{\chi}_{\perp}(T)$ have been calculated in a previous paper [14], based on the same spin model, which we used in the previous section.

In the following we model a nanowire of the size of about $197 \mathrm{~nm} \times 12 \mathrm{~nm} \times 12 \mathrm{~nm}$ with a cell size of $0.8 \mathrm{~nm}$. As before, along the long axis $(x)$ we apply a constant temperature gradient and fixed, antiparallel boundary conditions forcing a DW into the system. Figure 3(a) shows the position of the DW versus time for two different, microscopic damping constants $\lambda$. While for $\lambda=1$ the DW motion is smooth with constant tilting angle, for smaller damping constant the DW motion is accompanied by oscillations, indicating already that for
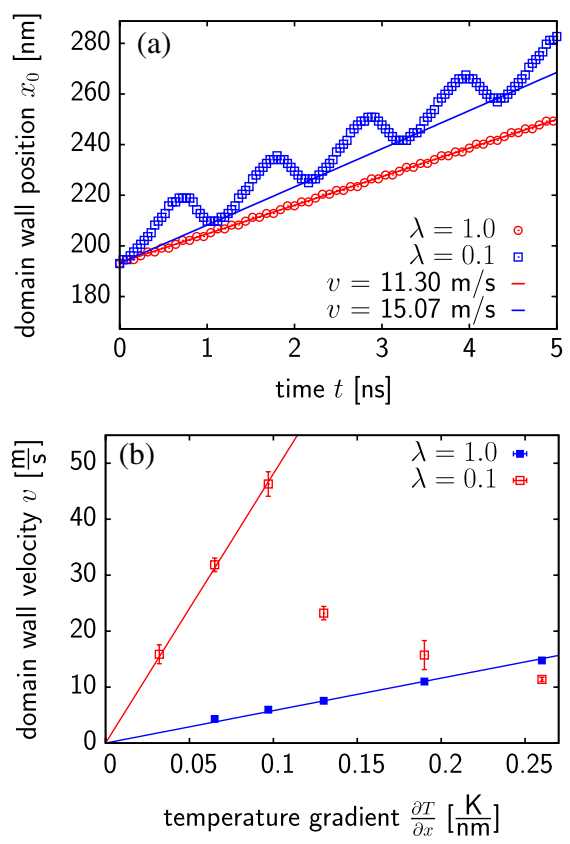

FIG. 3 (color online). (a) DW position versus time for two different temperature gradients. (b) DW velocity versus temperature gradient for two different damping constants $\lambda$. 
this new type of DW motion a Walker breakdown [19] exists [see discussion of Fig. 3(b)].

This DW motion is driven by the change of the effective fields of the LLB equation due to the temperature (i.e., space) dependent change of the thermodynamic properties of the DW. The most important role is played by the exchange fields. Following Eq. (3) the effective exchange field acting on one central spin in the DW is zero in equilibrium. Assuming that the system is now heated from one side, the magnetization vector on the hotter side shrinks. This gives rise to an effective exchange field acting on that central spin. This field has two components, one aligned with the magnetization vector, shortening its length, and one perpendicular to the magnetization vector. The latter part leads to additional torque terms in the LLB equation, which drive the DW motion. These torque terms correspond to the STT terms for DWs driven by spinpolarized currents [10-12], though striking differences exist. (i) Here naturally both terms, the adiabatic as well as the nonadiabatic term appear via the third and first term on the right-hand side of Eq. (2). Interestingly, the adiabatic term is smaller by a factor of $\alpha_{\perp}$. (ii) An additionally longitudinal term appears, which changes the length of the magnetization vector and with that the shape of the DW.

Figure 3(b) shows the DW velocity versus temperature gradient for different damping constants. Once again, in the high damping limit $(\lambda=1)$ the DW slides in a constant plane without any precession. In that case the DW velocity is proportional to the temperature gradient. In the case of the lower damping constant, the DW slides without precession until the Walker breakdown is reached. Above this threshold the DW motion is accompanied by precession and the DW velocity decreases, deviating from the linear behavior. The Walker breakdown comes from the fact that the plane defined by the magnetization rotation is pinned by the stray field of the DW (here to the $y-z$ plane). Above the Walker threshold the driving torque is strong enough so that the plane containing the DW is depinned and the DW starts rotating while it is moving. This pinning and depinning leads to the oscillatory motion of the DW observed in Fig. 3(a). A detailed quantitative investigation of the Walker breakdown is beyond the scope of this Letter but a similar behavior is found analytically $[11,12,20]$, numerically [21] as well as experimentally [22] for DWs driven by spin-polarized currents in the nonadiabatic case.

In conclusion, we have demonstrated that temperature gradients in a nanowire cause a DW motion via a magnonic SSE. Microscopically, this motion is due to a magnonic spin current where magnons move from the hotter into the cooler region. Because of the conservation of angular momentum, this spin current drags the DW into the hotter region. On a larger scale, magnons lead to a variation of thermodynamics quantities, which enter the exchange fields of a micromagnetic equation of motion - the LLB equation. These altered effective fields create spin torque terms, which drive the DW motion. Both pictures, the microscopic as well as the thermodynamic one, are in agreement. Nevertheless, one should note that in the thermodynamic picture magnons enter the equation of motion only effectively. Here, one has to assume that the magnetic system is locally in equilibrium, with the magnonic diffusion length smaller than the DW width. The DW velocities, which we forecast, can be of the order of $50 \mathrm{~m} / \mathrm{s}$. For lower damping constants this velocity could be achieved in temperature gradients much lower than those investigated here. Currently, we are not aware of any direct measurement of DW motion due to temperature gradient though a variation of the pinning fields of a DW in the vicinity of a laser spot was already measured [23]. Overall we expect this new type of DW motion to be measurable and, hopefully, to open the door for the development of spin caloritronic devices with new functionalities.

Funding from the Deutsche Forschungsgemeinschaft via Sonderforschungsbereich 767 is acknowledged.

[1] K. Uchida et al., Nature (London) 455, 778 (2008).

[2] C. M. Jaworski et al., Nature Mater. 9, 898 (2010).

[3] K. Uchida et al., Nature Mater. 9, 894 (2010).

[4] J. Sinova, Nature Mater. 9, 880 (2010).

[5] M. Hatami, G. E. W. Bauer, Q. F. Zhang, and P. J. Kelly, Phys. Rev. B 79, 174426 (2009).

[6] Y. Takezoe, K. Hosono, A. Takeuchi, and G. Tatara, Phys. Rev. B 82, 094451 (2010).

[7] Y. Kajiwara et al., Nature (London) 464, 262 (2010).

[8] J. Xiao, G. E.W. Bauer, K.C. Uchida, E. Saitoh, and S. Maekawa, Phys. Rev. B 81, 214418 (2010).

[9] J. C. Slonczewski, Phys. Rev. B 82, 054403 (2010).

[10] S. Zhang and Z. Li, Phys. Rev. Lett. 93, 127204 (2004).

[11] A. Thiaville, Y. Nakatani, J. Miltat, and N. Suzuki, Europhys. Lett. 69, 990 (2005).

[12] C. Schieback, D. Hinzke, M. Kläui, U. Nowak, and P. Nielaba, Phys. Rev. B 80, 214403 (2009).

[13] K. M. D. Hals, A. Brataas, and G. E. W. Bauer, Solid State Commun. 150, 461 (2010).

[14] N. Kazantseva et al., Phys. Rev. B 77, 184428 (2008).

[15] D. Hinzke et al., Phys. Rev. B 77, 094407 (2008).

[16] O. N. Mryasov, U. Nowak, K. Guslienko, and R.W. Chantrell, Europhys. Lett. 69, 805 (2005).

[17] U. Nowak, in Handbook of Magnetism and Advanced Magnetic Materials, edited by $\mathrm{H}$. Kronmüller and S. Parkin (John Wiley \& Sons Ltd., Chichester, 2007), Vol. 2.

[18] D. A. Garanin, Phys. Rev. B 55, 3050 (1997).

[19] N. L. Schryer and L. R. Walker, J. Appl. Phys. 45, 5406 (1974).

[20] R. A. Duine, A. S. Núñez, and A.H. MacDonald, Phys. Rev. Lett. 98, 056605 (2007).

[21] C. Schieback et al., Eur. Phys. J. B 59, 429 (2007).

[22] T. A. Moore et al., Phys. Rev. B 80, 132403 (2009).

[23] P. Möhrke et al., Solid State Commun. 150, 489 (2010). 